Du genre libertin au XVIII siècle, textes réunis par Jean-François Perrin et Philip Stewart, Actes du colloque international La Littérature libertine au XVIII ${ }^{\mathrm{e}}$ siècle: existe-t-il un genre libertin? Définition, typologie, limites chronologiques, corpus

\title{
Valentina Ponzetto
}

\section{(2) OpenEdition}

\section{Edizione digitale}

URL: http://journals.openedition.org/studifrancesi/27582

DOI: $10.4000 /$ studifrancesi.27582

ISSN: 2421-5856

\section{Editore}

Rosenberg \& Sellier

\section{Edizione cartacea}

Data di pubblicazione: 31 décembre 2006

Paginazione: 601-603

ISSN: 0039-2944

\section{Notizia bibliografica digitale}

Valentina Ponzetto, "Du genre libertin au XVIIIe siècle, textes réunis par Jean-François Perrin et Philip Stewart, Actes du colloque international La Littérature libertine au XVIIII siècle: existe-t-il un genre libertin? Définition, typologie, limites chronologiques, corpus », Studi Francesi [Online], 150 (L | III) | 2006, online dal 30 novembre 2015, consultato il 08 novembre 2020. URL : http://journals.openedition.org/ studifrancesi/27582; DOI : https://doi.org/10.4000/studifrancesi.27582

Questo documento è stato generato automaticamente il 8 novembre 2020.

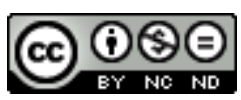

Studi Francesi è distribuita con Licenza Creative Commons Attribuzione - Non commerciale - Non opere derivate 4.0 Internazionale. 


\title{
Du genre libertin au XVIII ${ }^{\mathrm{e}}$ siècle, textes réunis par Jean-François Perrin et Philip Stewart, Actes du colloque international La Littérature libertine au XVIII siècle: existe-t-il un genre libertin? Définition, typologie, limites chronologiques, corpus
}

\author{
Valentina Ponzetto
}

\section{NOTIZIA}

Du genre libertin au XVIII siècle, textes réunis par Jean-François PERRIN et Philip STEWART, Actes du colloque international La Littérature libertine au XVIII ${ }^{\mathrm{e}}$ siècle: existe-t-il un genre libertin? Définition, typologie, limites chronologiques, corpus (Université Stendhal, Grenoble 3, 26-28 septembre 2002), Paris, Desjonquères, 2004, pp. 340.

1 La categoria critica di romanzo o racconto libertino è piuttosto recente: risale all'incirca alla metà del Novecento. Forse per questo, oltre che per il carattere ambiguo e multiforme della materia, il dibattito intorno alla definizione di genere libertino è stato negli ultimi anni assai vivo e acceso, non privo di note polemiche.

2 Il convegno internazionale tenutosi a Grenoble nel settembre 2002, di cui questo volume presenta gli atti, costituisce dunque un punto di riferimento fondamentale in materia. Per la prima volta i maggiori specialisti dell'argomento - ventitré studiosi venuti da sei paesi diversi - si sono riuniti per affrontare insieme, sotto i più diversi punti di vista, le delicate questioni della natura e della tipologia, dei limiti e delle 
suddivisioni di questo "genre nouveau consacré à l'amour sous toutes ses formes», un tempo considerato $d u$ second rayon e oggi sempre più alla ribalta. Dalle ricchissime comunicazioni e dai dibattiti che le hanno accompagnate sono emerse alcune conclusioni di grande importanza per gli studi futuri, prima fra tutte l'unanime accordo sull'effettiva esistenza del genere, con le sue costanti, il suo posto nelle coscienze dei lettori, la sua storia. Ne è specchio il significativo passaggio dalla formula interrogativa del convegno, existe-t-il un genre libertin?, a quella assertiva del volume, che preannuncia un dissertare intorno ad un nucleo comune indiscusso. Un accordo generale pare raggiunto sul corpus in analisi e sui suoi limiti cronologici, che corrispondono a grandi linee al XVIII secolo, fra Crébillon, indicato da molti studiosi come un iniziatore e un modello, e la Rivoluzione, o tutt'al più la Restaurazione. I suoi tratti più distintivi sono concordemente designati in una scrittura ellittica, più suggestiva che descrittiva, in sofisticate procedure di coinvolgimento del lettore, e in una costruzione letteraria sempre incentrata sulle arti del piacere, ma per lo più di una certa raffinatezza.

Restano forse da definire con più precisione le frontiere esatte del genere, all'esterno, soprattutto sul labile confine con la pornografia, ed eventualmente anche all'interno, fra diversi stili o tendenze. Fin dal Settecento, infatti, è stata avanzata una distinzione fra le categorie dell'ordure e del gazé, dell'osceno e del galante, il che dovrebbe probabilmente portare ad ammettere l'esistenza non di uno, ma di diversi generi libertini, e tuttavia la loro distinzione e definizione sistematica è impresa tutt'altro che semplice nell'ambito del rifiuto di ogni imposizione e di ogni limite tipica del libertinage, nonché ben lungi dall'essere universalmente approvata dai critici.

4 Proprio al più arduo dei compiti, «Définir», sono dedicati i saggi della prima delle tre sezioni in cui si divide il volume. Jean-Christophe ABRAMOVICI sceglie di risalire alle origini settecentesche della questione, analizzando, in Les Frontières poreuses $d u$ libertinage, le posizioni espresse da Pierre Bayle nell'Eclaircissement sur les obscénités (1701) e da La Mettrie in L'École de la volupté (1746), e concludendo per un'originaria divisione dei testi libertini in galants, délicats o voluptueux da una parte e obscènes o dissolus dall'altra. In linea con le più moderne teorie letterarie, invece, Peter CRYLE interpreta l'appartenenza ad un genere come un'autorizzazione di lettura iscritta nel testo. Il suo Le savoir-lire libertin è dunque volto a mostrare come i testi libertini si distinguano per la doppia regola di rifiutare ogni volgarità, ma al tempo stesso di non proclamare apertamente questo rifiuto, mantenendo una forma di ironica ambiguità. Come indica il titolo stesso, La fin du libertinage? di Michel DELoN, si preoccupa di definire i limiti estremi, non solo cronologici, del genere, contrapponendo sistematicamente l'estetica di Crébillon a quella di Nerciat, Louvet e soprattutto Sade in merito a quattro elementi essenziali della scrittura: i personaggi, i luoghi, i modi della narrazione e il rapporto con la Storia. Inversamente, Jean GoldzINK propone le sue Questions sur la naissance $d u$ récit libertin des Lumières, dove, dopo aver distinto nettamente e irrevocabilmente il «récit de moeurs libertin» dal «récit érotique ou obscène», si interessa esclusivamente al primo, individuandone il fondatore (Crébillon), i tratti salienti (amore mondano, moltiplicazione regolata e sistematica delle liaisons, rigorosa codificazione della lingua), e l'equilibrio fra tradizione e innovazione nel rapporto con $\mathrm{i}$ romanzi e i mémoires del Seicento. Philip STEWART, al contrario, si occupa delle diverse definizioni proposte per la letteratura pornografica o oscena. In Définir la pornographie? egli ne cataloga di quattro tipi, a seconda che guardino all'intenzione dell'autore, all'effetto prodotto sul lettore, al contenuto in quanto tale o ad una storia delle 
pratiche, finendo per privilegiare quest'ultima e mostrando come, a differenza di quanto sostenuto da Darnton, nel Settecento librai e polizia distinguessero una categoria di libri osceni che oggi definiremmo pornografici. Quanto a Jean-Pierre DUBosT, trovando troppo poco chiaro e distinto il concetto di letteratura libertina, che coprirebbe un corpus troppo eterogeneo, propone di sostituirlo con tre nuovi concetti presentati fin dal titolo del suo intervento: Erotologie, érotographie, libertinage. Il primo concernerebbe il discorso sulle cose del sesso, il secondo le forme di questo discorso e il terzo l'immagine prodotta da una mondanità libertina. Anche Caroline FISCHER sembra mal adattarsi alle terminologie già in uso e propone la categoria di «letteratura eccitante», cioè «qui excite la libido des lecteurs», riconoscibile per gli effetti prodotti sui loro corpi oltre che sulle loro immaginazioni. Il campo del romanzo libertino sarebbe delimitato per lei, in maniera quasi matematica, fra i due assi della portata filosofica e della carica eccitante (Littérature excitante et roman libertin). Infine Valérie VAN CRUGTEN-ANDRÉ in Le Roman du libertinage au tournant des Lumières riprende la sua definizione - già enunciata nella sua tesi del 1997 - di roman du libertinage, a suo dire più vasta e duttile di quella di roman libertin, e ne segue le evoluzioni storiche, tematiche e formali negli anni fra il 1782 e il 1815.

5 La seconda sezione, «Modéliser», risponde al tentativo di individuare all'interno del corpus riconosciuto per libertino dei modelli, dei topoi, che potrebbero rivelarsi distintivi del genere. Collection et oralité: de la réflexion sur la peinture au roman libertin, di René DÉMORIs mutua felicemente dalle riflessioni classiche sulla pittura i concetti di gusto e di collezione per applicarli ai fenomeni della lista e della circolazione degli oggetti del desiderio nel romanzo libertino. Questo rappresenterebbe dunque le donne come oggetti d'arte, laddove, inversamente, il collezionista d'arte tratta i quadri come oggetti d'amore. Pierre HARTMANN, in Nature, exemple, éducation: les paradigmes du récit libertin, interroga l'articolazione fra piano filosofico e piano narrativo nell'incontro fra $\mathrm{i}$ temi contradditori e tipicamente settecenteschi della natura e dell'educazione a partire da tre opere fondatrici (L'Académie des dames, L'école des filles, Vénus dans le cloître). $\mathrm{Ne}$ ricava un modello paradigmatico di racconto di formazione libertino in cinque tempi, di cui dimostra la pertinenza fino a Sade. Un altro archetipo narrativo, quello del «récit de séduction» nei romanzi libertini della mondanité, è presentato da Florence LOTTERIE (D'une loi du genre: les récits de séduction ou le libertinage mondain comme archétype narratif). Archetipo ambivalente, perché se da un lato si autodefinisce come ripetizione seriale delle stesse situazioni, repertorio, biblioteca di citazioni, dall'altro deve essere sempre singolare, sempre nuovo, proteiforme come il seduttore. Altri interventi seguono il ciclico ricorrere di un particolare motivo che si carica di significato fino a diventare emblematico della scrittura libertina. Così Dominique HöLZLE, con Les ambiguités du moment dans le roman galant, analizza minuziosamente le diverse manifestazioni di quella particolare disposizione dell'anima e dei sensi chiamata moment, mostrando come queste portino a galla le tensioni e le contraddizioni presenti nell'opera di Crébillon fra sentimenti e desiderio, discorsi intellettualizzanti e pura sensualità fisica, padronanza stilistica ed eccessi patetici; Catriona SETH, in Meubles-corps et corps-meubles; esamina la reificazione degli oggetti del desiderio secondo un processo di circolazione e alienazione dei corpi e degli oggetti che esprimerebbe i fantasmi erotici, le convinzioni materialiste e le angosce metafisiche del romanzo libertino fino a poter servire di metafora al genere intero; ed infine Patrick WALD LASOWSKI, con Coup sur coup trascina il lettore in una godibilissima cavalcata attraverso le occorrenze libertine delle 
espressioni a base di coups, fra sorprese improvvise, violenze, piaceri dei sensi. Jean SGARD, al contrario, mostra come in un unico romanzo, Le Sopha, siano racchiusi, come in una vera e propria «antologia critica» del genere, tutti i tipi di racconto che si possono ascrivere alla tradizione libertina: il racconto orientale, quello di metamorfosi, quello di piaceri sempre rinnovati, la carriera della prostituta e quella del seduttore e infine il romanzo di satira sociale.

6 La terza sezione, «Corréler», si propone di definire il genere libertino attraverso, l'intergenericità, l'altro da sé, il contatto o lo scontro con altri generi, altri sguardi vicini eppur diversi. In Libertinage et Mémoires secrets Christophe CAVE ha recensito quali definizioni danno di libertin e libertinage i Mémoires secrets attribuiti a Bachaumont, quali opere recensiscono e in che modo e quali invece passano sotto silenzio. Ne ricava un corpus relativamente ristretto e tendenzialmente bipartito fra opere à la manière de Crébillon e opere sulla scia del Portier des Chartreux, dunque in qualche modo fra il galante e l'erotico, nonché la constatazione che, negli ultimi anni del Settecento, le influenze fra letteratura $\mathrm{e}$ società sono reciproche $\mathrm{e}$ incrociate. Ai romanzi apertamente moraleggianti dei Toussaint, Lesbros de la Versane, Baculard d'Arnaud, Gérard, esatto contraltare del romanzo libertino, di cui spesso riprendono gli schemi, rovesciandoli, si è volto Henri COULET (Oeuvres en miroir: roman libertin et roman moral) dimostrando che, se non ci fossero, anche il romanzo libertino perderebbe forza fino a scomparire. I due generi, infatti, sono il prodotto e le due facce di una società retta da un ordine morale politico e religioso e armata di un organo di censura attivo ed efficace. In modo analogo e contrario Jean-François PERRIN, con L'Aliénation dans l'équivoque: licence et obscénité littéraires selon Rousseau, nota come il corpus di opere che oggi definiamo libertine offra una provocazione continua, addirittura una pietra di paragone al pensiero di Rousseau, ossessionato dall'alienazione dei segni linguistici che l'uso mondano ha irrimediabilmente pervertito, rendendo l'intera lingua francese passibile di equivoci osceni. Benoit MELANÇon esplora un legame particolare tra libertinage e romanzo epistolare analizzando i modi in cui può articolarsi, in alcuni romans par lettres del tardo Settecento considerati come libertini, il rapporto con la figura del «tiers inclus» - destinatario, editore fittizio, lettore virtuale o reale, sempre invitato alla partecipazione erotica nel senso più letterale del termine - chiedendosi poi se vi sia un rapporto necessario fra tale relazione triangolare e libertinage. Le altre comunicazioni completano la galleria di rapporti con i generi praticati nel Settecento: il petit genre della poesia erotica prediletta da Giorgio Baffo in Un libertin à la vénitienne: Giorgio Baffo (1694-1768) di Gérard LUCIANI; le memorie autobiografiche, intrise di modelli letterari e al tempo stesso più variegate, più complete e più accattivanti per il rapporto di continua seduzione che cercano di instaurare col lettore, in $D u$ «je» libertin di MarieFrançoise LUNA; il racconto, nella sua dimensione più ironica e sovversiva, che rimette in questione non solo ogni forma di autorità all'interno della narrazione, ma l'autorità stessa della narrazione, in Parodie et libertinage: le cas du roman-conte di Jean MAINIL; e ancora il teatro in Les Masques du séducteur: la carrière du libertin dans le théâtre du XVIIIe siècle di Pierre SAINT-AMAND, che vede nella scena un dispositivo dove si riproducono, enfatizzandosi, i comportamenti istrionici del frivolo petit-maitre e del diabolico roué già messi in scena dalla produzione romanzesca. 